Meta

Journal des traducteurs

Translators' Journal

\title{
Au dossier des métiers de l'informatique
}

\section{Robert Dubuc}

Volume 21, numéro 4, décembre 1976

URI : https://id.erudit.org/iderudit/002646ar

DOI : https://doi.org/10.7202/002646ar

Aller au sommaire du numéro

Éditeur(s)

Les Presses de l'Université de Montréal

ISSN

0026-0452 (imprimé)

1492-1421 (numérique)

Découvrir la revue

Citer cet article

Dubuc, R. (1976). Au dossier des métiers de l'informatique. Meta, 21(4),

259-260. https://doi.org/10.7202/002646ar d'utilisation que vous pouvez consulter en ligne.

https://apropos.erudit.org/fr/usagers/politique-dutilisation/ 


\section{DROBLEMES ET SOLUTIONS}

\section{AU DOSSIER DES MÉTIERS DE L'INFORMATIQUE}

Un texte récent ${ }^{1}$ consacré aux métiers de l'informatique laisse voir que le vocabulaire français de cette technique semble vouloir prendre ses distances à l'égard de la terminologie américaine. Naguère encore il n'était pas possible de risquer un équivalent pour hardware et software sans encourir les foudres des spécialistes. Cette mentalité semble en voie de régression si bien qu'on crée aujourd'hui des mots qui n'ont pas d'équivalents stricts en anglais. Tel est le cas du mot informaticien, si normalement tiré d'informatique. Les dictionnaires bilingues n'en finissent plus d'aligner les synonymes correspondant à cette notion : computer expert, computer specialist, computer scientist, data processing expert, specialist or scientist, ou plus familièrement $D P$ man. Cette pléthore synonymique ne fait que mettre en évidence l'absence d'un équivalent strict. Le mot informaticien est un terme générique désignant tous ceux qui se spécialisent professionnellement en informatique.

Il faut d'abord distinguer les informaticiens d'applications des informaticiens de systèmes ${ }^{2}$ : les premiers se préoccupent d'établir la programmation pour résoudre les problèmes particuliers de la clientèle (ils regroupent des ingénieurs ${ }^{3}$ (software engineer), des analystes ${ }^{4}$ (analyst) et des programmeurs 5 (programmer).

1. La terminologie française du présent article est tirée des “ Métiers de l'informatique ", dans Clartés, métiers et techniques, $\mathrm{n}^{\circ} 17$, Paris, novembre 1975. Les appellations de fonction $y$ sont exposées d'une façon rationnelle. C'est pourquoi il nous a paru intéressant d'en communiquer la teneur aux lecteurs de Meta.

2. Comme le mot informaticien n'a pas d'équivalent strict, il est normal que les locutions qui en dérivent n'en aient pas non plus. Pour rendre ces termes en anglais, ce sont encore les expressions computer applications specialist et computer systems specialist qui fourniraient les équivalents les plus satisfaisants. Ces fonctions sont souvent exercées par des ingénieurs d'où les expressions anglaises spécifiques applications engineer et systems engineer.

3. "Chargés de la conception des projets informatiques, les ingénieurs forment une catégorie diversifiée. Ils peuvent être spécialisés [...] dans les applications scientifiques, industrielles ou administratives pour software (logiciel) d'application.» Clartés, op. cit.

4. Les analystes ont pour rôle de traduire, de façon exhaustive et détaillée, les conceptions /de l'ingénieur $\%$. Id., ibid.

5. Les programmeurs sont chargés de rédiger dans un langage compréhensible par la machine les instructions qui feront exécuter $[\ldots]$ les opérations décrites dans l'analyse. Id., ibid. 
Les informaticiens de systèmes sont ceux qui connaissent parfaitement les caractéristiques et les possibilités des machines et peuvent à cet égard conseiller les informaticiens d'applications, qui travaillent en général sous la direction d'un chef de projet (head of project).

Si l'on passe maintenant à l'exploitation, on trouve d'abord sous le chef d'exploitation (supervisor of operations) le personnel de saisie (input staff), qui a pour rôle d'alimenter le monstre ordinateur. Il comprend entre autres les perfodactylos (tape-punch operator) qui transcrivent sur bandes de papier perforées les informations à traiter, les perforateurs ou mieux perforeurs ${ }^{6}$ (card-punch operator) qui portent l'information sur cartes perforées, les encodeurs ${ }^{7}$ (magnetic encoder operator) qui transcrivent l'information sur bandes magnétiques, enfin le pupitreur (computer operator) assure la " conduite " de l'ordinateur par l'intermédiaire d'un pupitre de commande. L'alimentation de la machine est confiée à des opérateurs périphériques (peripheral operator) qui montent les bandes magnétiques sur les dérouleurs de bandes, chargent les disques dans les tourne-disques ou surveillent le fonctionnement des imprimantes.

\section{RÉSUMÉ TERMINOLOGIQUE}

analyst

card-punch operator

computer applications

computer expert, specialist, scientist

computer operator

computer systems specialist

data processing expert, spcialist, scientist

DP man (fam.)

head of project

input staff

magnetic encoder operator

peripheral operator

programmer

software engineer

supervisor of operations

tape-punch operator

\author{
analyste \\ perforeur \\ informaticien d'applications \\ informaticien \\ pupitreur \\ informaticien de systèmes \\ V. computer expert \\ V. computer expert \\ chef de projet \\ personnel de saisie \\ encodeur \\ opérateur périphérique \\ programmeur \\ ingénieur logiciel \\ chef d'exploitation \\ perfo-dactylo
}

ROBERT DUBUC

6. Ces fonctions étant le plus souvent remplies par des femmes, ce sont les formes féminines perforeuse, encodeuse qu'on rencontre le plus souvent.

7. Le mot encodeuse désigne aussi l'appareil. 